\title{
Tactile Toys: Therapy for Tactile Dysfunctions
}

\author{
Karen Hong \\ Singapore Institute of Technology, Singapore
}

\begin{abstract}
Tactile Toys are developed from threedimensional textiles as tactile therapy for children who are diagnosed with Tactile Dysfunction. The three-dimensional forms of textiles are achieved by utilizing the thermoplastic qualities of synthetic fabrics. Tactile Toys are being used in occupational therapy sessions, and the usage of specific Tactile Toys for each individual child is closely being monitored by the occupational therapist. The implementation of Tactile Toys as tactile therapy will better equip children with appropriate therapeutic tactile sensory benefits, with a focus in helping children with different tactile sensory needs to improve their ability to regulate, interpret and execute appropriate behavioural responses to touch sensations. The goal of tactile therapy for children is to help them develop appropriate responses to touch sensation so that daily tasks can be competently performed. As these competencies will increase with effective tactile therapy, the child's self-esteem and self-regulation will also improve, allowing them to live their lives in a functional manner.
\end{abstract}

\section{Introduction}

Victoria is a five-year-old girl who is extremely sensitive to the types of clothes she wears and textures that she comes into contact with. The bedding, towels, upholstery and even the carpet must be of a certain level of comfort to her. If she cannot find her comfortable socks and if she walks on the carpet with her bare foot, it will trigger her frustrations and lead to a meltdown. In contrast, seven-year-old Jason generally seems unaware and unbothered by textures of any surfaces, unlike Victoria who is extremely sensitive. On the other hand, Aaron loves to touch and feel everything! He craves for certain textures and will touch, feel, stroke, poke or pull anything that comes into his sight. These three children do not react to the sense of touch as normally as compared to other children.

The above-mentioned three children are diagnosed with Sensory Processing Disorder. They are known to have tactile sensory dysfunction. To help them cope with this dysfunction, a series of tactile toys and tactile materials collectively known as Tactile Toys are designed and prototyped for these children. This paper focuses on the conception of Tactile Toys and their usage as tactile therapy to help children with tactile sensory dysfunction to overcome their dysfunction.

Tactile Toys will help expose the children to different types of touch sensations within textiles that they will come into contact with, such as their play objects or daily necessities that they use regularly. Children who are oversensitive or under-responsive to the sense of touch as well as tactile-seeking will benefit from this range of play objects and materials. Tactile Toys will further attract children to explore and enjoy tactile experiences. The three-dimensional structural surfaces can respond to the child's sense of touch and sight. They are incorporated as sensory diets during the occupational therapy sessions facilitated by an occupational therapist that will be monitoring the appropriate use of tactile toys and tactile materials for the child with specific tactile sensory dysfunction.

Through tactile therapy, their tactile defensiveness can be improved, their low level of sensitivity to textures can be enhanced and their tactile seeking behaviours can be soothed. Tactile therapy will allow them to develop appropriate responses to touch sensations in their daily lives.

\section{Literature Review}

In this review, the effectiveness of occupational therapy on children with sensory processing disorder will be discussed. Occupational therapist practices the Sensory Integration Approach on children and it is important to review how therapy is being administered for children and specific therapy methods used for these children. Next, the usage of generic textiles as therapy and the current textilebased materials used for tactile therapy for children with tactile dysfunction will be reviewed. Subsequently, the fundamentals of using the heat setting technique to achieve three-dimensional structural qualities on fabrics, which is a vital feature 
in the design of Tactile Toys, will also be reviewed. The use of human computer interaction technology in special needs therapy also contributes to the claim of this research. Finally, the convergence of all the above research areas resulting in the conception of Tactile Toys will summarize how Tactile Toys can contribute to the existing tactile therapy and eventually allow the children to overcome the dysfunction and respond appropriately to tactile senses in their daily lives.

\subsection{Occupational Therapy for Children with Sensory Processing Disorder}

In the $3^{\text {rd }}$ European Congress of Sensory Integration 2014, researchers share their research results and knowledge of sensory integration and sensory processing disorder. Current research and knowledge got its start from research by the late Dr Jean Ayres. Ayres was both an occupational therapist and an educational psychologist who developed sensory integration therapy for children with Sensory Processing Disorder. Till today, there are still ongoing research to look into the effectiveness of the sensory integration approach and how this approach has helped children to overcome the disorder.

Founder of Sensory Processing Disorder Foundation, Dr Lucy Jane Miller, one of the key presenters at this congress has published numerous evidence-based researches on the effectiveness of occupational therapy using the sensory integrated approach for children. Miller et al [1] conducted a controlled trial on three groups of children with sensory processing disorder. First group of children received the occupational therapy-sensory integration approach, second group received activity protocol and the third group no treatment at all. The children's behaviour, sensory and adaptive functions were closely administered. The findings showed that the group who received the occupational therapy-sensory integration approach has improved tremendously in terms of the child's sensory responsivity, social behavior, motor competence, and participation in meaningful daily activities. Sensory integration approach do ameliorate difficulties of children with sensory processing disorder, which supports the claim of this research whereby the design of appropriate tactile sensory materials with three-dimensional surface textures can assist children to overcome specific reactions to tactile sensations through incorporating these toys into the sensory diets of their occupational therapy.

Teresa A. May-Benson, Executive Director at the Spiral Research Center and Laboratory is a wellknown expert in sensory integration in occupational therapy and known clinically for her work with children and adults with sensory processing problems. This center is dedicated to expanding the body of research on sensory processing. May-Benson together with Koomar [2] reviewed the effectiveness of sensory integration approach on the ability of children with sensory processing disorder to engage in desired daily activities. Results suggest the sensory integration approach may result in positive outcomes in sensorimotor skills and motor planning, socialization, attention and behavioral regulation, reading-related skills, participation in active play and finally achievement of individualized goals. The findings have shown positive trends supporting the effectiveness of the sensory integration approach, especially when measuring goals customized for the child. This review suggests that there is a trend towards positive evidence to support the sensory integration approach.

Associate Professor, Beth Pfeiffer from the Department of Rehabilitation Sciences at Temple University, Philadelphia, United States did a more focused study on children with Autism Spectrum Disorder who has sensory processing difficulty. Pfeiffer et al [3] test out the effectiveness of sensory integration approach on a group of children between ages six to twelve. These children were randomly put into two groups; the first group received fine motor skills therapy and the second group sensory integration therapy. The children's social responsiveness, sensory processing, functional motor skills and social-emotional reactions were closely tracked before, during and after the therapy. The findings showed significant positive changes for both groups; but even more significant changes occurred in the group who received sensory integration therapy with significant decrease in their autistic mannerisms.

The reviews of sensory integration approach of occupational therapy do support the claim of this research, which is to design appropriate tactile sensory materials with three-dimensional surface textures that can assist children to overcome specific reactions to tactile sensations through incorporating these toys into the sensory diets of their occupational therapy. Sensory-based strategies using the right products in an occupational therapy session can help in the improvement of a child's ability to tolerate or make sense of sensory input. The review concludes that sensory integration approach indeed shows evidence that children do respond to sensory integration positively by reducing the child's behavioural responses to environmental stimuli, modulating of child's cortisol levels as well as allowing better school and social participation. The focus is to provide the child with opportunities to experience sensory inputs at just the right level that he can tolerate, along with engaging him in sensory

\footnotetext{
i 3rd European Congress of Sensory Integration, ESIC2014. http://www.congress.utu.fi/esic2014/index.php
} 
activities that will help calm or alert him. By using this approach, the child begins to accept and respond more appropriately to play and daily activities and hence allowing him to live his life in a functional manner.

\subsection{Textiles as Therapy - Current Textile- based Therapeutic Products}

In the occupational therapy practice guidelines published by Renee Watling et al, sensory-based therapy will provide a child with vestibular, proprioceptive, auditory and tactile inputs in the form of different activities. [4] These activities can help the child to organize their sensory system and improve their sensory processing skills. Specially designed equipments and materials can provide the right sensory input for the child. A typical occupational therapy treatment room looks like a play gym. There are suspended swings, therapy balls, mats, tactile materials, and developmentally appropriate toys. The child will be lead by the occupational therapist to work on different types of equipment during the session and activities are adapted depending on the child's acceptance and behaviour.

Specifically for children with tactile dysfunction, there are textile-based toys, materials and equipments that are utilized in the therapy sessions. In another pilot study conducted by Professor Sandra Dunbar et al, [5] tactile experiences are also embedded around classrooms and playgrounds. These materials often incorporate a variety of textures made from different types of fabrics, plastics, papers, feathers and other textured materials. Different tactile sensations are also being explored by asking the child to find 'treasures', which are very often small toys hidden in a tank of sand, pasta, rice, shaving cream or Play-Doh. Occupational therapists are aware of the individual child's tactile dysfunction, from tactile overresponsive to under-responsive to sensory seeking and will plan which activities to work with the child accordingly.

There are specific textiles based therapeutic products available commercially. One good example is the 'weighted vest'. Associate Professors Jennifer Stephenson and Mark Carter from Macquarie University, Australia; reviewed the use of weighted vests on children with autism spectrum disorders and other disabilities. Weighted vests are being used during sensory integration therapy as an intervention strategy and may assist in remediating problems such as inattentiveness, hyperactivity, stereotypic behaviors and clumsiness. [6] In another pilot study on the effectiveness of weighted vests, Collins and

\footnotetext{
ii "A Comforting Design: K-State Students Design, Produce Vests for Children with Autism." (2011) News Services, June 27. https://www.k-state.edu/media/newsreleases/jun11/ autismvest62711.html
}

Dworkin focused on the use of weighted vest on children with attention difficulties. Weighted vests are widely considered to be a sensory-based approach because they are intended to provide specific sensory input to improve performance and function. The weight of the vest provides proprioceptive input, which is defined as deep pressure and promotes production of neurotransmitters such as serotonin and dopamine, which modulate the activity of the central nervous system. [7]

In Kansas State University, apparel design students had a special project to design and produce special vests for children with autism or sensory integration disorders. The small weights on the vests are strategically placed so they can be felt on different points of the body; provide a calming effect to children so they can focus on a particular task. There are several pockets for weights and the vests provide a tight fit to help the child to be calm and focus. Recycled or donated fabrics are used to make the vests, and the students ensure that they select materials that would be comfortable for the children and also easy to maintain. Extra design elements, such as buttons are added for the child to practice fine motor skills. ${ }^{\text {ii }}$

In Singapore, T.Ware is a start-up company who has created a jacket that could 'hug' its wearer. The research and technology behind the T.jacket started at the research lab of the Keio-National University of Singapore Connective Ubiquitous Technology for Embodiments Centre. The T.jacket looks like an ordinary jacket, but it has three pairs of inflatable air bags placed at the shoulders, abdomen and lumbar regions of the jacket. All are connected to a tiny motor box at the back. Users can download an app into their smartphones and use it to control the pressure levels of the individual airbags. The smartphone remote controller allows a single caregiver or therapist to control multiple jackets with different pressure levels. The T.jacket mimics deep-pressure stimulation, such as hugs, used by therapists to calm children when they get overexcited. As explained by Mr James Teh, the chief executive of the company, the unique feature of the jacket is the ability to alter the pressure levels and it is the key to the effectiveness of the jacket because different children respond better to different levels of 'hugs'. iii

In a study done by McGinnis et al, [8] they investigated the effects of using deep pressure touch as a therapy method when occupational therapists are utilizing the sensory integration approach. When an individual is hugged, squeezed, stroked, or held, a sense of deep pressure touch is produced. In occupational therapy interventions, activities

\footnotetext{
iii "Cashing in on the Calming Power of Hug Therapy." (2012) The Straits Times, Apr 11. http://newshub.nus.edu.sg/news/1204/
} PDF/HUG-st-11apr-p3.pdf 
associated with deep pressure are frequently used. For example, occupational therapists often use equipment such as mats, pillows, beanbag chairs, mattresses, trampolines, trapeze bars, chin-up bars, and climbing structures that are thought to provide deep pressure stimulation. The results of this study suggest that the activities occupational therapists use to administer deep pressure can improve the proprioceptive sense for children with autism and related spectrum disorders. These deep pressure activities are advantageous for several reasons. The materials involved, such as blankets, therapy mats, or cushions, are typically a one-time, low-cost investment. These materials are easily accessible in many home and community settings and are a healthy alternative to oral medications given to the children.

The reviews of current textile-based sensory products do support the claim of this research, which ascertains the design of textile-based sensory toys and materials. The three-dimensional textiles created for this research are collectively known as Tactile Textiles. These fabrics are created by using the heat setting techniques and are designed into Tactile Toys. These newly created sensory toys will be integrated as sensory diets within a child's tactile therapy session. This review reinforces that sensory integration approach is successful with the incorporation of specific equipments, toys and textiles-based materials, all rightfully prescribed into sensory activities that can assist children to overcome specific reactions to tactile sensations. By using this approach, the child will be provided with the sensory experiences he needs to feel more balanced and comfortable in his life.

\subsection{Heat Setting to Create Three-dimensional Textiles}

The integral part of Tactile Textiles is the design and creation of the three- dimensional structural forms of textiles. These three- dimensional structural textiles are formed by using the heat setting techniques which is a technical approach of fabric manipulation that provides a platform into some of the most creative and innovative approaches to surface and textile design. The design of Tactile Toys from Tactile Textiles with three-dimensional surface textures can assist children to overcome specific reactions to tactile sensations through incorporating these toys into the sensory diets of their occupational therapy.

In order to achieve three-dimensional textures on a fabric, the technique of heat setting is applied. Professor Sara Kadolph from Iowa State University, College of Human Sciences; also author of 'Textiles' [9] has defined that heat setting is a process that uses heat to stabilize the shape and dimensions of fabrics. The fabric is heated to bring it to a temperature specific for the fiber being heat set. At this temperature, the fiber molecules move freely to relieve stress within the fiber. Tension remains until the fiber has cooled, locking this shape into its molecular structure. After cooling, the fabric will be stable to any heat lower than that at which it was set.

An active researcher in the field of Textile Design and specialist in resist patterning for contemporary fabrics, Dr Kate Wells author of 'Fabric Dyeing and Printing' [10] explains that heat setting of all synthetic fibres will melt at a certain temperature, but below this point they will often heat set into a different form. Tying, stitching or clamping fabrics into folds before heating can create various features. One hundred percent polyester fabric works best with heat setting. Polyester belongs to the group of synthetic fibres. Polyester is thermoplastic, that is, it can be transformed through heat into new configurations, which on cooling is completely stable. The definition of thermoplastic by Braddock and O'Mahony [11] states that the molecular structure of the fiber breaks down and becomes fluid at a certain temperature, making it possible to reshape the fabric by pleating, moulding, vacuum-forming or crushing. The fabric is 'fixed' on cooling and cannot be altered unless heated to a temperature greater than the one at which it was reshaped. This three-dimensional surface structure is hence permanent and it can be maintained even with washing in cold water.

Professor Sherry Haar from the Department of Apparel, Textiles and Interior Design of Kansas State University [12] defines heat setting as the creation of three- dimensional textures on fabrics through shaped-resists. During this process, heat has been applied to stabilize the shape of the fabric. This technique is achieved by mass production in an industry and designers have also adapted it by using non-industrial heat setting methods. Haar has further researched into the other possibilities of shape and resist techniques and categorized the methods of resist as binding, stitching, clamping and pole wrapping [12]. There are endless possibilities of shaping fabric to create three-dimensional structures. Methods can be combined and adapted, unique heat setting forms can also be developed through utilizing interesting shapes as moulds.

The three-dimensional structures on the textiles can be attained by applying 'shibori' techniques. President of World Shibori Network, Yoshiko Iwamoto Wada author of 'Memory on Cloth: Shibori Now' defines shibori as a variety of ways of embellishing textiles by shaping cloth and securing it before dyeing. [13] The areas that are secured will resist the dye and when released, beautiful dyed patterns are formed on the cloth. However, in the heat setting of three-dimensional structures, fabrics are securely tied to moulds or manipulated before putting into the steamer or boiler. The resulting threedimensional effects will be very effective and the shape will be permanent. The heat set fabrics can be 
hand wash in cold water and the shapes will still be stable.

Wada [13] further elaborates the technical production of polyester and how polyester fabric reacts to heat setting. Invented in 1940 s, polyester is made up of chemical composition of oil, ethylene glycol and terephthalic acid. It comprises of linear molecules of carbon, hydrogen and oxygen bonded together. This bond can be broken by heat and hence be shaped in response to heat. Heat is crucial in the production of polyester filament. During the extrusion process, melted polyester is extruded through tiny holes and stretched to many times its original length that changes the inner structure of the material to provide strength and stability. The stretched filament is then heat set at a temperature below its melting point. When this degree of heat is applied to polyester fabric, it reverts to its un-stretched state, shrinking drastically. Polyester's softening point is about $240^{\circ} \mathrm{C}$ and its melting point is $260^{\circ} \mathrm{C}$. The ideal temperature for polyester fabric to be heat set to achieve a threedimensional structure is around $200^{\circ} \mathrm{C}$ to $220^{\circ} \mathrm{C}$. The time taken for heat setting should be between 20 to 30 minutes for ideal results.

Dr Rachel Philpott [14], a British textiles designer and researcher explains that contemporary pleating and shibori practices have been transformed by the advent of thermoplastic materials like polyester, which have shape memory capabilities allowing permanent folds to be created. Her area of research concentrates on the development of high performance textiles and she creates adaptable, self-supporting three-dimensional textile structures with shape memory materials applicable in sportswear, medicine, architecture, interior and product design.

Designers of textiles, fashion and costume have explored the shaping and heating of fabrics as an aesthetic feature. The creative process can also be applicable to various creative interiors and lifestyle products. This range of heat set fabrics, with its tactile quality; not only draws the attention of one's eyes but the hands too and the textures can stimulate our sense of touch. With its multiple functionalities, they can be hung on walls or under a ceiling as sculptures; be framed as screens; as curtains; as cushions and light shades. Heat set Tactile Textiles can be extremely versatile in its form and is very suitable to be developed into Tactile Toys and tactile materials. These three-dimensional forms not only conjures the imagery of the natural elements but also intrigues and attracts the audience to touch and feel the structural forms. With this appealing characteristic, it will provide a variety of tactile sensations and explorations using touch and therefore generate more interest for the children. This will better equip the children with the appropriate therapeutic tactile sensory benefits and hence focus on helping children who are over or under-responsive to step out of their fear of touching textures.

\subsection{Human Computer Interaction Technology in Special Needs Education}

Researchers in the field of Human Computer Interaction (HCI) have designed and developed touchbased therapy for children with autism and sensory processing disorders. One example is the design of haptic interfaces developed at the MIT by Vaucelle et al [15]. They have presented their findings at the SIGCHI Conference on Human Factors in Computing Systems in April 2009. They have created devices that stimulate touch through haptic devices to help clinical therapy; which are non-invasive treatments. They named the prototypes "Touch Me", "Squeeze Me", "Hurt Me" and "Cool Me Down" and have tested these devices with a team of mental health professionals. Their devices incorporate smart technology and are designed to be 'cause and effect' play objects, these play objects allows the child to understand that an action they do can cause a respond from this device. The embedded technology will include motion sensors, which will detect hand movements on the surface of the fabrics, and other embedded technology such as vibrotactile, pneumatic and heat pump actuation. These devices served as tools for the therapist to communicate better with children and also providing therapeutic comfort to them. They have been successful, as all the prototypes have been evaluated for further development and are used as complementary therapeutic aids.

The development of touch-based therapeutic products in field of Human Computer Interaction has seen success in combining smart technology and therapy for children with autism and sensory processing disorder. The convergence of these main areas of research give rise to the conception of Tactile Toys and will be the contribution of this research project. A tactile dysfunctional child who is under responsive, over responsive or sensory seeking will be attracted to the three-dimensional surface textures and bright colours of the Tactile Toys. Tactile therapy through the use of Tactile Toys will provide a variety of tactile sensations and explorations using touch. This will better equip the children with the appropriate tactile sensory reflexes and hence focus on helping them to overcome their dysfunction and allow them to respond appropriately to tactile senses.

\subsection{Conception and Contribution of Tactile Toys}

As supported by several reviews of researchers in the field of sensory integration therapy, sensory integration approach enhances a child's sensorimotor and motor planning skills, improves his socialization, attention and behavioral regulation, reading-related skills, and participation in active play. [2] Positive trends are supporting the effectiveness of the sensory 
integration approach, especially when measuring goals customized for the child. This is further supported by the analysis for outcomes of occupational therapy using a sensory integration approach from parents done by Cohn et al. [16] Parents hope that with early intervention, their child can improve in their self-regulation, participation in skilled motor activities and self-confidence.

Tactile Therapy consisting of textile-based toys, materials and equipments that are utilized in the therapy sessions and other textiles related therapeutic products such as the weighted vest, compression vest and compression shirts has also shown positive and effective results to help the child overcome tactile dysfunction. This review reinforces that sensory integration approach is successful with the incorporation of specific equipments, toys and materials, all rightfully prescribed into sensory activities that can assist children to overcome specific reactions to tactile sensations. Therefore supporting the claim of this research, which ascertains the design of textile-based sensory toys and materials.

The contribution of the research is to create original Tactile Textiles that has three-dimensional surface structures. Tactile Textiles will be further designed into tactile toys and tactile sensory materials and will be integrated as sensory diets within a child's tactile therapy session, coordinated by an occupational therapist. Collectively known as Tactile Toys, the unique three-dimensional surface textures can assist children to overcome specific reactions to tactile sensations through incorporating these toys into the sensory diets of their occupational therapy.

Tactile Toys will be beneficial and a valuable contribution to the special needs community specially targeted at children with tactile dysfunction. Current textiles based toys utilized in the therapy sessions are mainly normal two-dimensional textured fabrics with no special textile treatments or manipulations. The creation of the three-dimensional textiles by heat setting has not been applied as a therapy method within the needs for the child with tactile dysfunction. Different versions of Tactile Toys targeting respective tactile needs will be included into the sensory treatment sessions of the occupational therapy, allowing the child to learn and become accustomed through exposure of these toys and materials. Hence, Tactile Toys will be able to fill up the gaps of the existing tactile therapeutic toys, materials and equipments used in occupational therapy for children with sensory processing disorder.

\section{Design Concept: Tactile Toys as Tactile Therapy for Children with Tactile Sensory Dysfunction}

Touch is the most basic of human senses. The tactile sense provides information through the surface of our skin and allows us to receive information regarding the texture, shape and size of objects in our environment. [17] This is known as the discriminative factor of the tactile system. With a functioning sense of touch, we can feel pressure, temperature and pain, which is the protective factor of the tactile system. Protective factors alert us to potential dangers. [18] Being able to react positively to touch sensations enables us to feel comfortable and emotionally secure.

Tactile Toys are a series of play objects and materials designed for children with Tactile Sensory Dysfunction. They are made from specially designed three-dimensional fabrics collectively known as Tactile Textiles, which are constructed by utilizing the thermoplastic qualities of synthetic fabrics. These three-dimension structural textiles are formed by using heat setting techniques, which is a technical approach of fabric manipulation that provides a platform into some of the most creative and innovative approaches in surface and textile design. This technique enables a flat fabric to be transformed into structural and sculptural forms. The creative process of pleating, crushing as well as moulding continues to evolve into different possibilities and hence creating a range of interesting surface designs that is fundamental to the design process of this project.

The main choice of fabric material is polyester, chosen because the three-dimensional structures should be able to retain their shapes permanently. Polyester, with thermoplastic qualities in the form of fabrics and fibres can be given a three-dimensional form regardless of construction methods and amalgamation with different surface design techniques. By definition, thermoplastic refers to the quality of a fibre whose molecular structure breaks down and becomes fluid at a certain temperature, [19] making it possible to reshape the fabric by pleating, moulding or crushing. The fabric is 'fixed' on cooling and cannot be altered unless heated to a temperature greater than the one at which it was reshaped.

Polyester belongs to the fabric group called Synthetic Fibres. Some other examples in the synthetic fibre group include Polyamide, Acetate, Acrylic, Viscose and Elastane. Polyester is thermoplastic, that is, it can be transformed by heat into new configurations, which on cooling are completely stable [11]. Polyester fibres and fabrics, being thermoplastics can be given a new form by heat setting. In this project, different techniques of heat setting will be explored to show the flexibilities of Polyester being given a three-dimensional structure and form.

Moulding of the fabric creates structural surfaces on the fabric. Moulds of different shapes and sizes can be used to provide the three-dimensional effect of the fabric. One easy approach is to apply simple 'shibori' techniques, by using a binding technique; glass marbles can be tied to the polyester organza. Then 
fabric can be placed into a microwave or steamer during the heating process. Once heated, the fabric will adhere to the form of the marble mould, which is below the melting point of the fabric. Once the fabric has been heat set, the marbles are removed. This creates an amazing textural, rounded and threedimensional effect to the fabric. This structural design can be customized to one's liking, whether the moulds are close together or further apart, each individual fabric can be created with a different form.

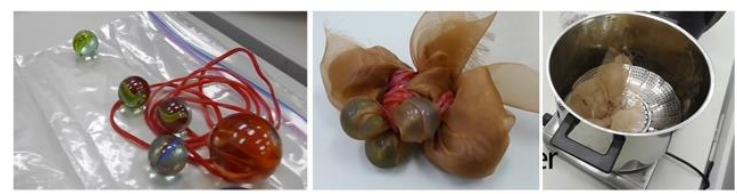

Figure 1. Tying of marbles and heating to achieve three-dimensional effect on fabric

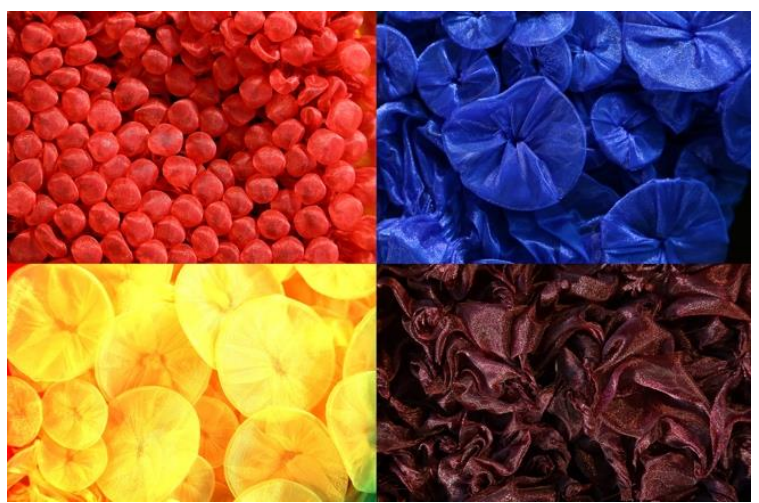

Figure 2. Tactile Textiles: Various shapes achieved by using different moulds

Shibori is a centuries old traditional Japanese textile finishing technique. It involves tying and folding a piece of fabric before the dyeing process. This technique is originally used on silks and plant fibres, which leads to unique patterns, textures, structural forms and colours after the dyeing process. On natural fibres, the three- dimensional effect will not be permanent. However, on synthetic fibres such as polyesters, when treated with the shibori technique and heat, the fabrics will have a permanent threedimensional surface as a result.

Tactile Textiles are hence being developed into a range of tactile sensory products that target children who suffer from tactile sensory dysfunction. It aims to target children with tactile sensory dysfunction that involve the tactile sense who may be suffering from either over-responsiveness, under-responsiveness or sensory-seeking disorders. A child who is sensory over-responsive overreacts to their sensory messages. Examples of symptoms include being highly irritable to fabric textures particularly those that are rougher such as denim or wool. If left untreated, the child may grow up to face problems in grooming, as combing of hair, nail cutting, hair cutting will become highly irritable. Their senses are too sensitive to withstand such activities, which to them has become undesirable. A sensory under-responsive child displays slowed response towards a sensory message. Some symptoms include not crying when hurt, not noticing when someone touches him, not reacting to different body sensations such as heat, cold, and hunger. Some children may also be socially withdrawn. Lastly, a sensory-seeking child is fidgety and will be constantly touching or pulling something because he has a large sensory appetite. Constantly touching something or being fidgety is an attempt for him to satisfy his sensory cravings.

The treatment for tactile sensory dysfunction is through an occupational therapist. A sensory integration approach will be adopted. According to the child's symptoms, a customized sensory diet will be planned to treat the child. Some examples of existing treatments [19] include squeezing soft tactile balls, use of Willbarger's brush to apply pressure and proprioception and, doing art and craft that involves different textures such as clay and paint.

Tactile therapy through the use of threedimensional structural textiles will provide a variety of tactile sensations and explorations using touch. The implementation of tactile sensory products into the sensory diets of the children will better equip them with the appropriate therapeutic tactile sensory benefits, and hence is able to focus on helping children with different tactile sensory needs to improve their ability to regulate, interpret and execute appropriate behavioural responses to touch sensations so that they are able to live their lives in a functional manner. The goal of tactile therapy for children is to help them develop appropriate responses to touch sensation so that daily tasks can be competently performed. As these competencies will increase with effective tactile therapy, the child's self-esteem and self-regulation will also improve.

\section{Proof of Concept: Effectiveness and Value of Tactile Toys}

Tactile Toys are woven into the sensory diets of children who are sensory over-responsive, sensory under-responsive and sensory-seeking. The treatment for each specific tactile dysfunction is through an occupational therapist. According to the child's symptoms, a customized tactile sensory diet is planned as treatment for the child. The four Tactile Toys, Tactile Touchables, Tactile Balls, Tactile Runway and Tactile Wrap are prototyped; user studies are conducted to proof the concept. The proof of concept here is to test out the effectiveness of the Tactile Toys in a real life situation. These four toys are brought to an occupational therapy center, for duration of three weeks. With the help of the occupational therapists, a child from each category of tactile dysfunction is selected for the test. This is needed to examine how effective tactile toys are for 
children with tactile dysfunction.
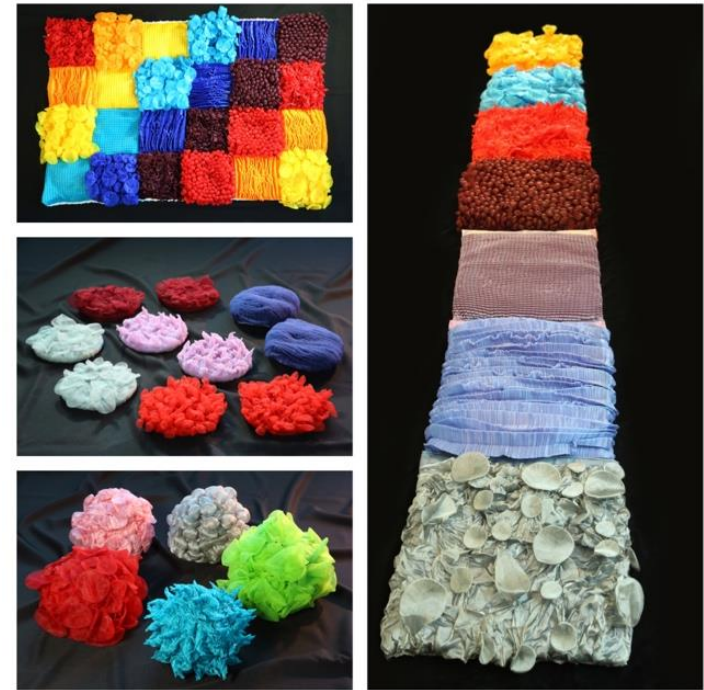

Figure 3. Collection of Tactile Toys: Tactile Wrap, Tactile Touchables, Tactile Balls and Tactile Runway

For children who are sensory-seeking, tactile toys will provide them with sufficient sensory cravings and the cause-and-effect play would capture their attention so that they can sit still long enough to go through the sensory therapy. For sensory overresponsive children, Tactile Toys will allow them to practice the feel and touch of different types of fabrics so that they will become more comfortable in feeling the different textures over time. As long as the child has been exposed to a variety of touch sensations, the uncomfortable feel that she has initially will gradually diminish. This will be the strategy of desensitization [20], which is a gradual process whereby the child is introduced to a variety of textures and materials and over time, they will learn to accept the tactile sensation. As for sensory under-responsiveness children, they will be able to benefit from the tactile and visual stimulation provided by the tactile toys and materials. The invigorating three-dimensional structures and the bright vivid colours together with the cause-and-effect play catches the children's attention and interest, while allowing them to maintain sufficient focus on the toys.

It is important to introduce tactile experiences slowly and gradually and only when the child is ready to experience them, so that any defensive reaction can be avoided. A child with tactile defensiveness should never be forced to touch anything they do not want to, as this will cause further apprehension and avoidance. The therapist will need to encourage, explain, understand and communicate with the child as they attempt to introduce touch sensations to them in a safe and non-threatening way. If the child with tactile defensiveness is fearful of any materials, the therapist will let the child play with it in a less threatening way.
For example, the child can hold the therapist's hand to start touching the tactile toys. The child can put objects or toys inside or outside the Tactile Toys, or push his or her toy cars or plush toys through it. After a child begins to feel safe, he or she will be slowly encouraged to try other fun ways in which the therapist can demonstrate, such as poking or pulling the Tactile Toys. Eventually, the child will be encouraged to use their whole hand, including their palm to touch the tactile textures, or even roll their body over it.

A summary of comments given by each therapist working with their selected child was as follows. For the therapist working with a sensory over-responsive child, she felt that Tactile Touchables and Tactile Runway served the purpose of as a Tactile Toy that can help children to improve on tactile discrimination. These Tactile Toys worked well when they were being used in a constructive game context. Combining Tactile Toys with other sensory activities that can help with the other senses of the body such as visual, proprioceptive or vestibular and using Tactile Toys to enhance fine or gross motor skills of the child would be advantageous. For example, when Tactile Touchables were being used as a matching game, it would improve visual-spatial awareness, proprioception and motor planning of the child. When the child moved her hands from left to right, she would be working on her ability to cross the body midline, which was a skill important for smooth movement. Her body movements of picking and putting down the discs from right to left could improve proprioception and body awareness. Her ability to match the discs in pairs and tracking the therapist's motion could boost her visual motor skills and hand-eye coordination. Being able to organize her body to do what she needed to do would enhance her motor planning skills. The ability to observe and respond to the therapist's motions could develop gestural communication. In this way, the therapy would be effective, entertaining and stimulating for the child.

As for the therapist working with the sensory under-responsive child, she felt that Tactile Toys could provide tactile stimulation for a tactile underresponsive child. As the child had to receive more sensory messages in order to wake up his under responsive systems, the therapist needed to provide frequent, varied and extended opportunities for tactile stimulation, together with deep pressure and vestibular input to alert his sensory systems. Hence, Tactile Toys will need to be combined with the other existing therapy equipments within the play gym to provide maximum benefits for the child. The vivid colours of the Tactile Toys could stimulate his visual sense, the three dimensional textural structures provided him with fresh sensations and tactile stimulation. With lots of movement involving proprioceptive and vestibular inputs, it would enable 
him to increase his alertness in his daily lives and be able to perform his daily tasks normally.

The therapist who worked with the sensoryseeking child commented that Tactile Toys could provide tactile stimulation to satisfy his tactile cravings. Tactile Toys can be incorporated into his daily living environment so that he can use these toys routinely which provides him with tactile stimulation. As he needs access to fidget toys and tactilely diverse materials, Tactile Balls and Tactile Touchables can help him to satisfy his cravings for touch stimuli. When he is over-aroused, the Tactile Wrap can serve to calm him down together with deep pressure provided by his caregivers. Tactile Toys can be used to support appropriate behaviour, increase his attention and organization skills and prevent overstimulation. Within the occupational therapy environment, Tactile Toys works well when combined with the other existing therapy equipments and planned play activities. The toys together with the other existing therapy equipments and planned play activities will provide the sensory craving child the appropriate proprioceptive, vestibular and tactile input to modulate his sensory system. In this way, he will be able to engage in fine or gross motor activities in school and will be more prepared to learn and focus as well as to keep him organized.

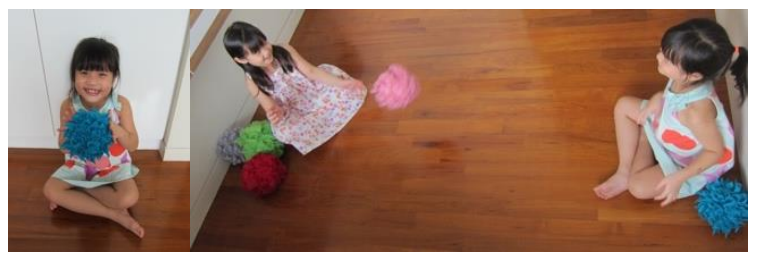

Figure 4. Playing "Toss and Catch" with Tactile Balls

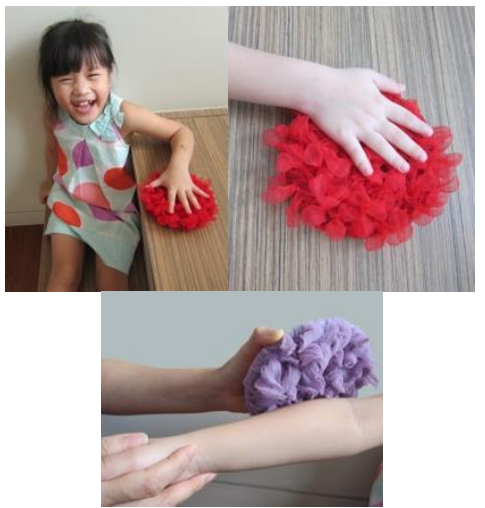

Figure 5. Feeling and stroking the textures on Tactile Touchables and brushing of Tactile Touchables on the child's hand

Overall, Tactile Toys have proofed to be effective when they are handled well and strategically used by occupational therapists. With its intriguing textures and structural forms, Tactile Toys attract children to touch them. With the help and supervision from occupational therapists or parents, children can manipulate and explore these toys by rubbing, feeling, squeezing and kneading using their hands and having their hands in contact with Tactile Toys in various planned activities and games.

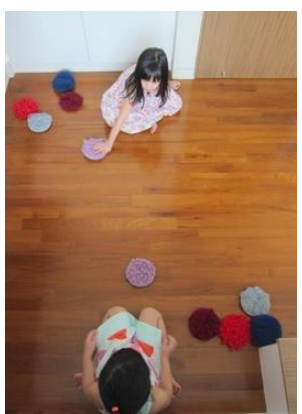

Figure 6. Playing matching game using Tactile Touchables

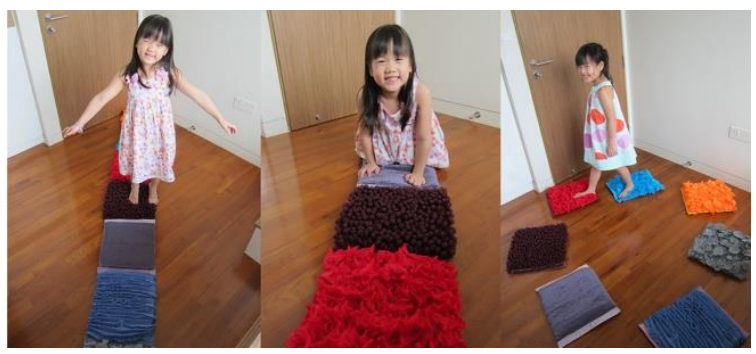

Figure 7. Walking, crawling and using Tactile Runway as "Step Upon" mats

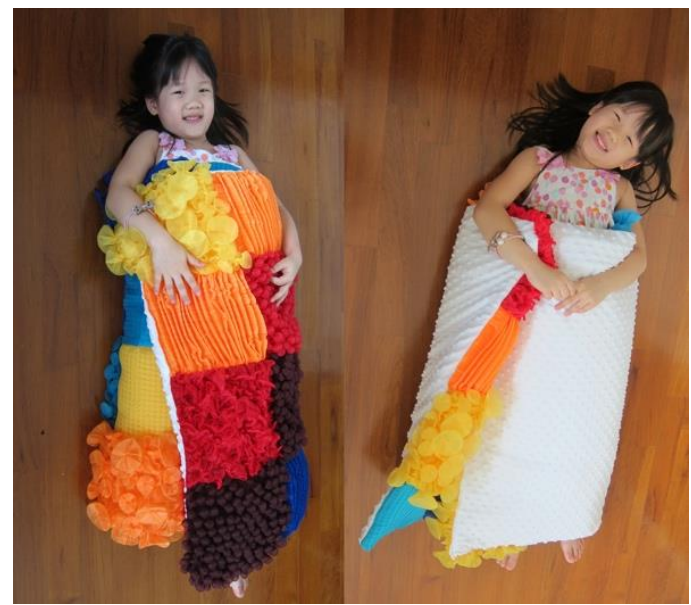

Figure 8. Playing "Burrito" using Tactile Wrap

On the whole, Tactile Toys do stimulate children's tactile, proprioceptive, vestibular and visual senses as they offer the children a rich sensory play experience. Tactile Toys will heighten the children's awareness of their body, allowing them to feel their physical and kinesthetic sensation within their body. This may make them more in tuned with their physical self and they will eventually step out of their tactile dysfunction behaviours. 


\section{Conclusion}

Tactile Toys are designed to help children with tactile dysfunctions. With the detailed study of children suffering from sensory processing disorder focusing on types of tactile dysfunctions; the technical understanding of the construction methods of threedimensional textiles and the thorough study of sensory integration approach, Tactile Toys are created as a means of tactile therapy. Tactile therapy through the use of three-dimensional structural textiles will provide a variety of tactile sensations and explorations using touch. The implementation of threedimensional structural forms into Tactile Toys will generate more interest for the children and focus on helping children who are over-responsive, underresponsive and sensory seeking to acquire the appropriate tactile sensory inputs for their tactile dysfunctions. This will better equip the children with the appropriate therapeutic tactile sensory benefits and hence focus on helping children to improve their ability to regulate, interpret and execute appropriate behavioural responses to touch sensations so that they are able to live their lives in a functional manner. The goal of tactile therapy for children is to help them develop appropriate responses to touch sensation so that daily tasks can be competently performed. As these competencies will increase with effective tactile therapy, the child's self-esteem and self-regulation will also improve.

Tactile Toys can calm children and regulate their stress levels. When working with their hands, the kinesthetic hand movements such as pressing and squeezing can help children to release their physical energy or tension. [21] These experiences are important for the children to build up their emotional well-being and regulate their sense of emotions. When combined with guided activities and games, Tactile Toys can engage children's fine and gross motor skills. The children will be able to control their motor movements during directive play, which will prove beneficial in improving their self-control. Occupational therapists will know when to increase the challenges of the activities when children have accomplished certain levels and this will allow children to feel successful and elevate their sense of achievements. This will greatly help to strengthen their self-esteem and increase their confidence level in performing their daily activities. Hence allowing them to step out of their tactile dysfunction and to be more competent and independent in allowing them to live their life to the fullest.

\section{References}

[1] Miller, L. J., J. R. Coll, and S. A. Schoen. (2007) "A Randomized Controlled Pilot Study of the Effectiveness of Occupational Therapy for Children With Sensory
Modulation Disorder." American Journal of Occupational Therapy61, no. 2: 228-38.

[2] May-Benson, T. A., and J. A. Koomar. (2010) "Systematic Review of the Research Evidence Examining the Effectiveness of Interventions Using a Sensory Integrative Approach for Children." American Journal of Occupational Therapy64, no. 3: 403-14.

[3] Pfeiffer, B. A., K. Koenig, M. Kinnealey, M. Sheppard, and L. Henderson. (2011) "Effectiveness of Sensory Integration Interventions in Children With Autism Spectrum Disorders: A Pilot Study." American Journal of Occupational Therapy65, no. 1: 76-85.

[4] Watling, R. (2011) Occupational therapy practice guidelines for children and adolescents with challenges in sensory processing and sensory integration. Bethesda, MD: American Occupational Therapy Association.

[5] Dunbar, S.B., Carr-Hertel, J. et al. (2012) "A pilot study comparison of sensory integration treatment and integrated preschool activities for children with autism." The Internet Journal of Allied Health Sciences and Practice 10 (3): 1-8.

[6] Stephenson, J. and Carter, M. (2009) "The use of Weighted Vests with Children with Autism Spectrum Disorders and Other Disabilities." Journal of Autism and Developmental Disorders 39 (1): 105-114.

[7] Collins, A. and Dworkin, R.J. (2011). "Pilot Study of the Effectiveness of Weighted Vests." The American Journal of Occupational Therapy 65 (6): 688-94.

[8] McGinnis, A. A., Blakely, E. Q., Harvey, A. C., Hodges, A. C. and Rickards, J. B. (2013). The behavioral effects of a procedure used by pediatric occupational therapists. Behavioral Interventions, 28(1), 48-57.

[9] Kadolph, S.J. (2010). Textiles. $11^{\text {th }}$ ed. Upper Saddle River, NJ:Prentice Hall.

[10] Wells, K. (1997). Fabric Dyeing \& Printing. Conran Octopus Limited.

[11] Braddock, S.E., and O’Mahony, M. (1998). Techno Textiles. London: Thames \& Hudson, Limited.

[12] Haar, S.J. "Studio practices for shaping and heat-setting synthetic fabrics." (2011). International Journal of Fashion Design, Technology and Education 4, no. 1 : 31-41.

[13] Wada, Y.I. (2012). Memory on Cloth: Shibori Now. New York: Kodansha International.

[14] Philpott, R. (2012) "Crafting innovation: The intersection of craft and technology in the production of contemporary textiles." Craft Research 3(1) 53-74.

[15] Vaucelle, C., Bonanni, L., \& Ishii, H. (2009). "Design of haptic interfaces for therapy." In Proceedings of the SIGCHI Conference on Human Factors in Computing Systems, pp. 467-470. ACM.

[16] Cohn, E. S., Kramer, J., Schub, J. A., \& May-Benson, T. (2014). Parents' explanatory models and hopes for 
outcomes of occupational therapy using a sensory integration approach. American Journal of Occupational Therapy 68, no.4: 454-462

[17] Kranowitz, C. S. (2006). The out-of-sync child has fun: activities for kids with sensory processing disorder. Perigee Book.

[18] Auer, C. R., \& Blumberg, S. L. (2006). Parenting a child with sensory processing disorder: a family guide to understanding and supporting your sensory-sensitive child. Oakland, CA: New Harbinger.

[19] Miller, L. J., Fuller, D. A., \& Roetenberg, J. (2014). Sensational kids: hope and help for children with sensory processing disorder (SPD). NY, NY: A Perigee Book.

[20] Mays, J. H. (2011). Your childs motor development story: understanding and enhancing development from birth to their first sport. Arlington, TX: Sensory World.

[21] Snyder, C. (2011) Providing Sensory Experiences That Meet the Needs of All Infants and Toddlers. http://www.highscope.org/file/NewsandInformation/ Extensions/ExtVol25No5_low.pdf. 